\title{
S-NICS Investigation for Heterocyclic Anticancer Compounds
}

\section{NAYYER T. MOHAMMADIAN, KARIM ZARE and MAJID MONAJJEMI*}

\author{
Department of Chemistry, Science and research Branch, Islamic \\ Azad University, Tehran, Iran. \\ ${ }^{*}$ Corresponding author Email: m_monajjemi@srbiau.ac.ir
}

http://dx.doi.org/10.13005/ojc/330402

(Received: June 06, 2017; Accepted: July 14, 2017)

\begin{abstract}
There are no works in theoretical of a statistical method of NICS-nucleus independent chemical shift for study of heterocyclic rings, since the asymmetry ${ }^{15}(\eta)$ and $\operatorname{skew}^{15}(\kappa)$ parameter is fluctuated in small distance and are alternate in large distance in the center of heterocyclic rings . Through changing the asymmetries among $0 \leq \eta \leq+1$ skew $^{15}$ could be changed in the range of $-1 \leq \kappa \leq+1$, and also the parameter $\kappa$ will be zero when $\sigma_{22}=\sigma_{\text {iso }}{ }^{15}$. In position of axially-symmetric tensors, $o_{22}$ equals ${ }^{15}$ either $\sigma_{11}$ or $\sigma_{33}$, skews are $\kappa= \pm 1$. In this study, we have investigated the statistical methods through nucleus-independent chemical shifts-SNICS calculations in view point of $\mathrm{Bq}$ motions in the center of sphere spaces of heterocyclic rings. In our previous work ${ }^{15}$, it has been exhibited that S-NICS method is an accurate method for estimation the amount of aromaticity in the non-benzene ${ }^{15}$ rings similar heterocyclic rings which are popular molecules in organic chemical compounds as anti-cancer disease. Although NICS values for benzene and naphthalene and so on can be indicated as aromaticity criterion, for other molecules such as heterocyclic rings and their derivatives, S-NICS values are much more accurate compare to NICS index.
\end{abstract}

Keyword: S-NICS, NICS, heterocyclic anticancer compounds

\section{INTRODUCTION}

Cancer is a disease dating as far back as the dinosaurs has found cancerous lesions on dinosaur bones. Egyptians also drew examples of breast cancer in their hieroglyphics on papyrus, and by the 4th century B.C. many types of tumors such as stomach and uterine cancer had been described. In 1775 Percival Pott, a London physician, linked the incidence of scrotal cancer in men to their jobs as chimney sweeps when they were young boys. It was not, however, until the 19th century that scientists began to study cancer systematically, looking at what causes cancer and how it can be cured.

Cancer is one of the important causes of death in the new century. This work is done for developing of modern anticancer drugs. Many of heterocyclic compounds are known as anticancer drugs such as alkylating agents which have targeted 
cell DNA causing cell death. Heterocycles ${ }^{1}$ structures are composed by atoms other than carbon, where the most times substituents are sulfur, oxygen ${ }^{1}$ and nitrogen ${ }^{1,2}$.The model size of heterocycles ring, together with the substituent group of the core scaffold ${ }^{2}$, impact tightly on the chemical and physical properties ${ }^{3}$ while among the clinical applications, heterocyclic compound has an active role as anti-bacterial ${ }^{4,5}$, anti-viral ${ }^{6}$, anti-fungal ${ }^{7}$, antiinflammatory ${ }^{8}$ and anti-tumor drugs ${ }^{9-11}$.

Among the heterocyclic compounds the pyridine, thiophene and Thiazole derivatives exhibited large amount cytotoxicity towards the cancer cell lines. Structure activities relationship was reduced ${ }^{9}$ from biological results and will be used in further design ${ }^{11}$ of new active compounds. Currently, a number of drugs are used in the treatment ${ }^{11}$ of the cancer, but most of them were produced controlled effect on the cancer cells. The usual applications of heterocycles are as vast as it is diverse and is not extensively ${ }^{9}$ encompassed in the scope $^{10}$ of that study.

The most drugs belong to a class of heterogenius structures. Heterocyclic structures played an important behavior in the metabolism ${ }^{11}$ of all cells; maximum number of them is 6 (or sometimes 5) membered hetero-cycles including one to three heteroatoms ${ }^{11}$. Recently, imidazole fragment has been attracting much concentration because of its role as attractive scaffold for biochemical active heterocyclic ${ }^{11}$ drugs $^{12}$.
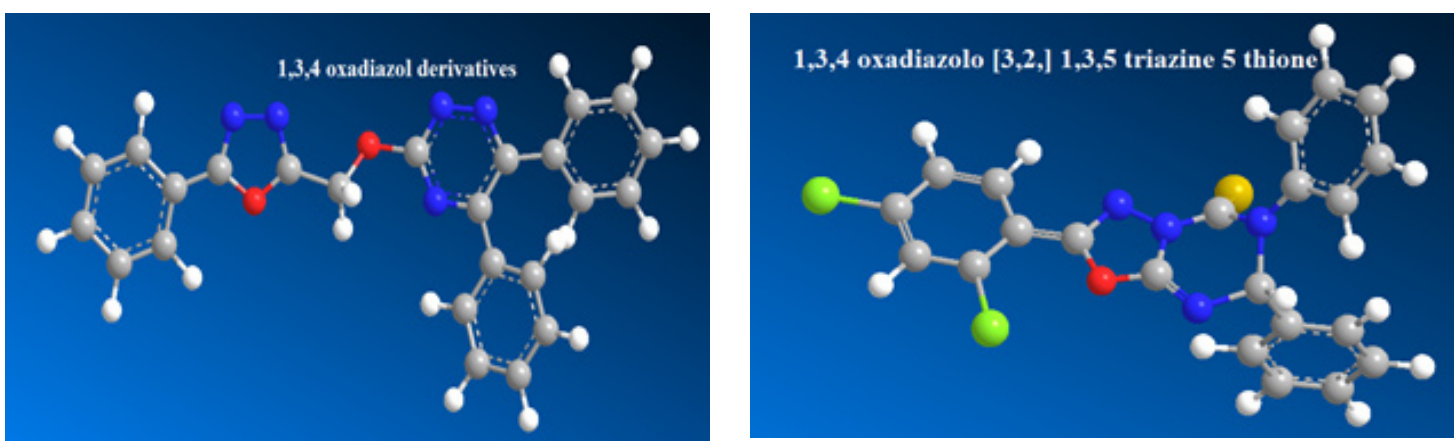

Fig. 1: optimization of 1,3,4 oxadiazol derivatives and 1,3,4 oxadiazolo [3,2,] 1,3,5 triazine 5 thione

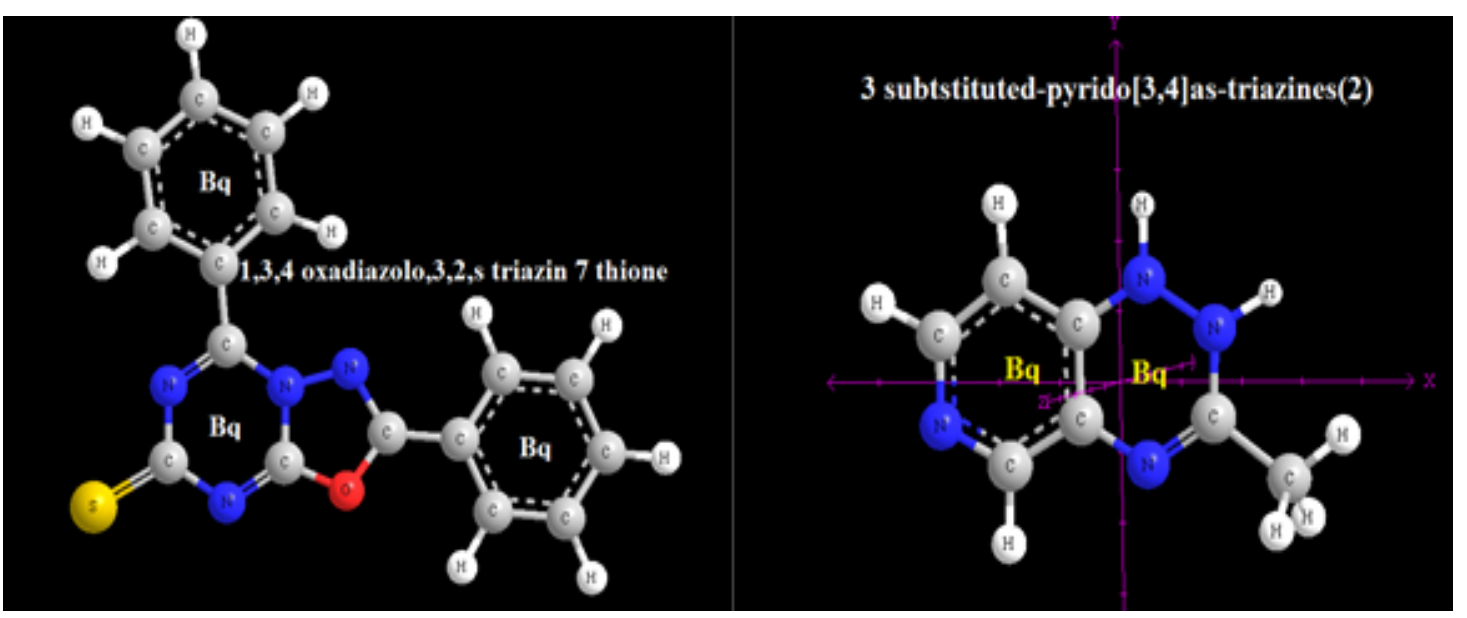

Fig. 2: optimization of 1,3,4 oxadiazolo,3,2,s triazin 7 thione and 3 subtstituted-pyrido[3,4]as-triazines(2). 
Generally, chemical-physics and biochemical properties like acceptor and donor capabilities, hydrogen bond, $\pi-\pi$ interactions, van der Waals, coordination ${ }^{12}$ bond with a metal and in total hydrophobic force has caused much interest in anticancer studies for such compounds. These properties are important of understanding for its reactivity enable derivative for binding with various nucleic acids, enzymes and biological structures ${ }^{13,14}$.

A large number of the important heterocyclic compounds are used in the medical activities such as histidine and proline which are amino acids. It is notable pyridoxine, folic acid, thiamine, riboflavin,

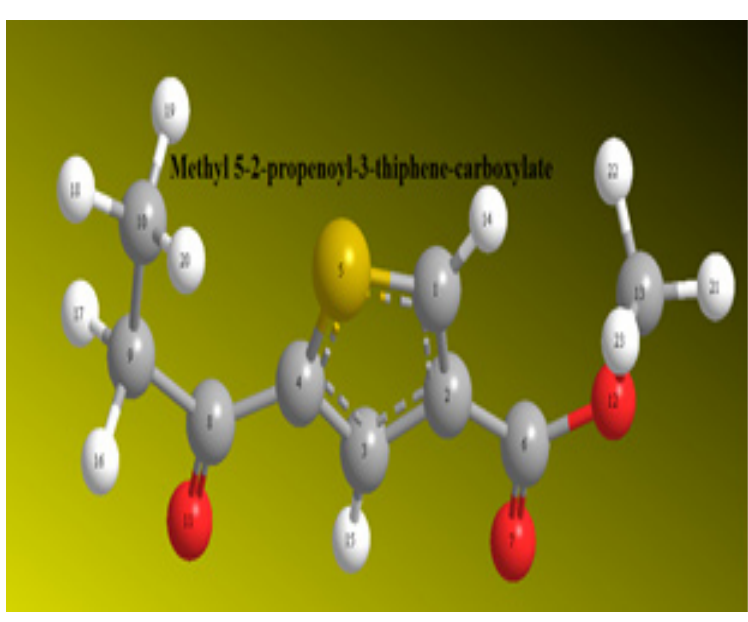

biotin, $B_{12}$ and $E$ families of the vitamins are included of heterocyclic structures. For investigation of antifungal activity compounds, Singh et al have synthesized 1,3, 4oxadiazolo-(3,2a)-s-triazin7-thione ${ }^{13,14}$ and Abdle,et-al have synthesized some novel 1, 3, 4oxadiazole derivatives ${ }^{12,13}$. Fungi ${ }^{13}$ are hetero-tropic micro-organisms that are distinguished $^{13}$.

Dhar has synthesized 1,3,4-oxadiazolo[3,2-a]-1,3,4-dithiazines and found anti-fungal. In compound $\mathrm{Ar}=2-\mathrm{ClC} 6 \mathrm{H} 4, \mathrm{Ar}^{\prime}=2-\mathrm{ClC} 6 \mathrm{H} 4 \mathrm{OCH}$. Methyl 5-( I-hydroxy-2-propenyl)-3-thiophenecarboxylate was stirred at room temperature with 10 equivalents of freshly prepared manganese dioxide to give

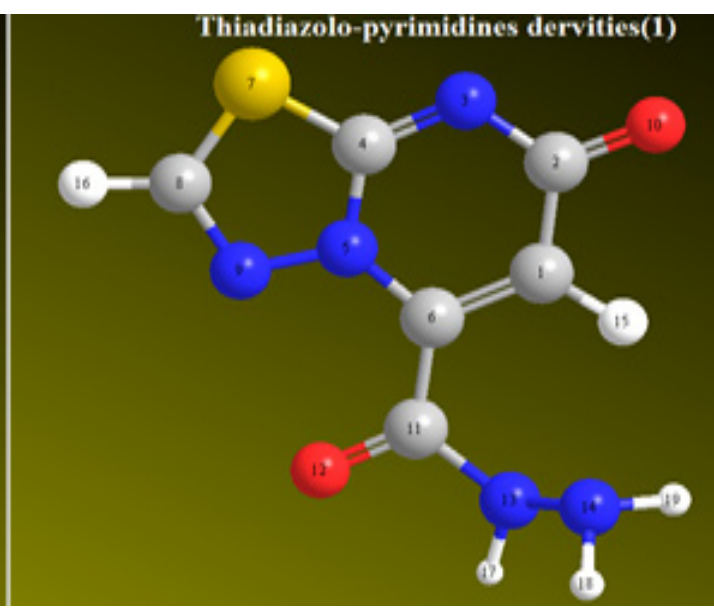

Fig. 3: optimization of Methyl 5-2-propenoyl-3-thiphene-carboxylate and Thiadiazolo-pyrimidines derivatives (1)
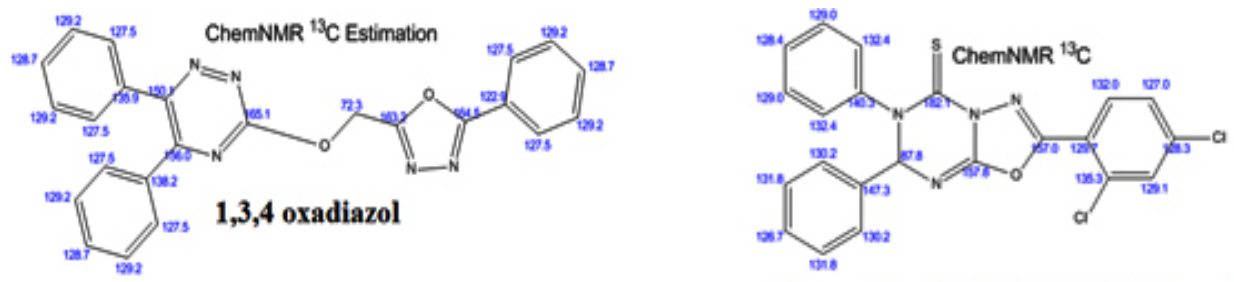

$1,3,4$ oxadiazolo $[3,2] 1,3,$,5 triazine 5 thione
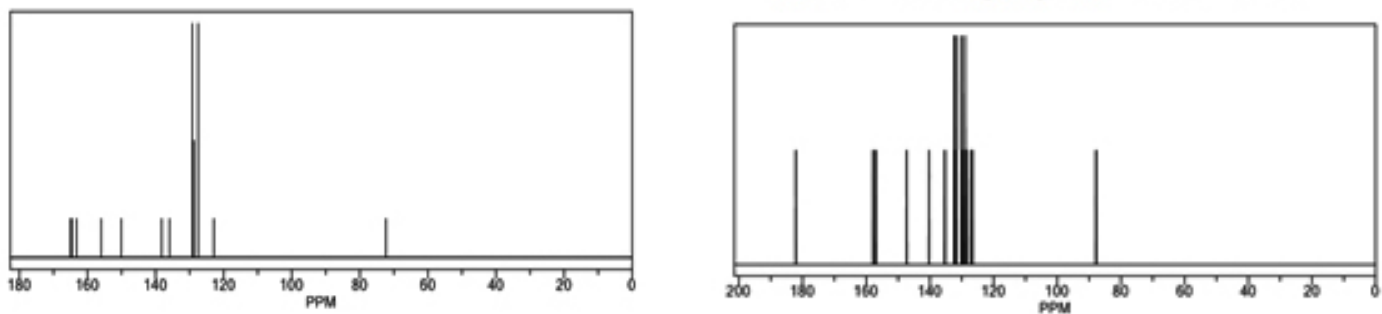

Fig. 4: NMR estimation of some oxadiazol compounds 

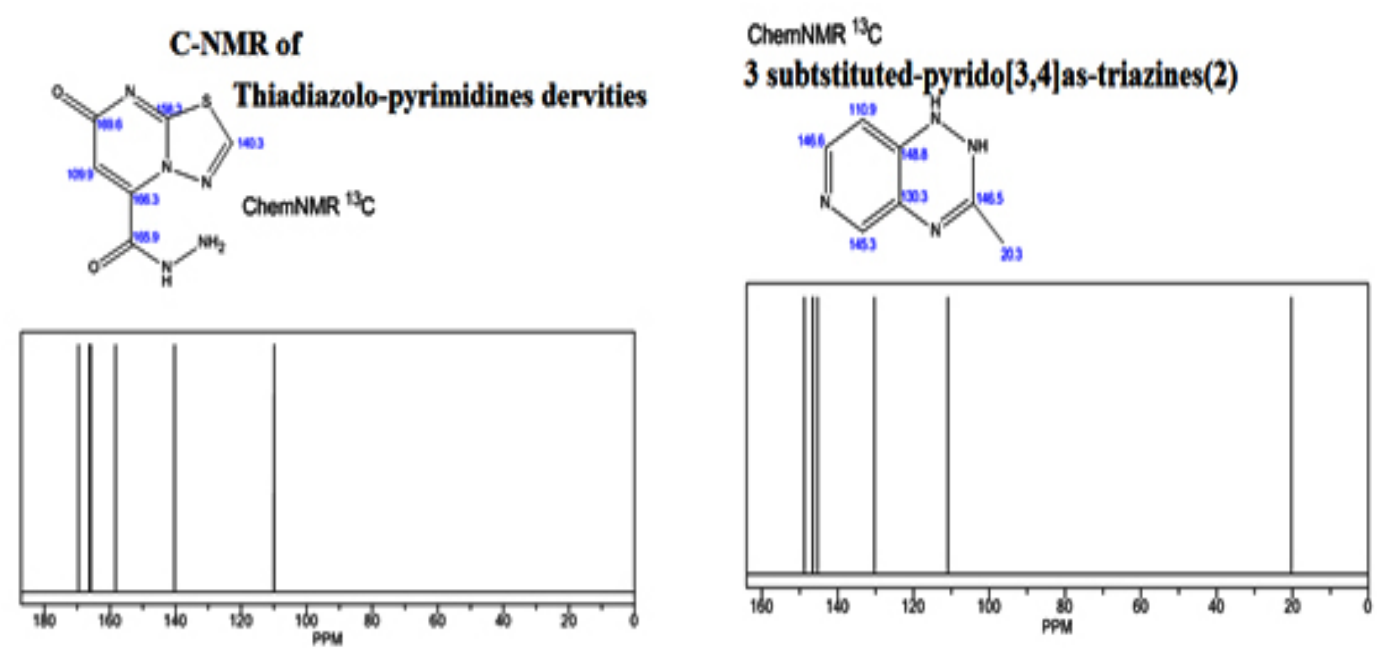

Fig. 5: NMR estimation of some Thiadiazol compounds

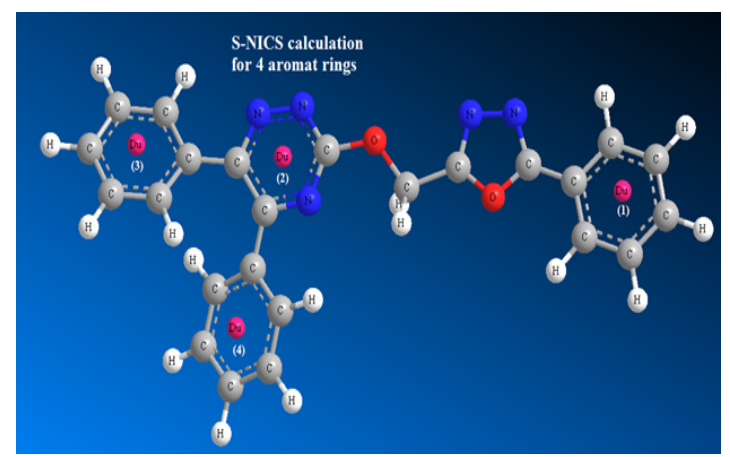

Fig. 6: S-NICS calculation for 4 ring through dummy atoms

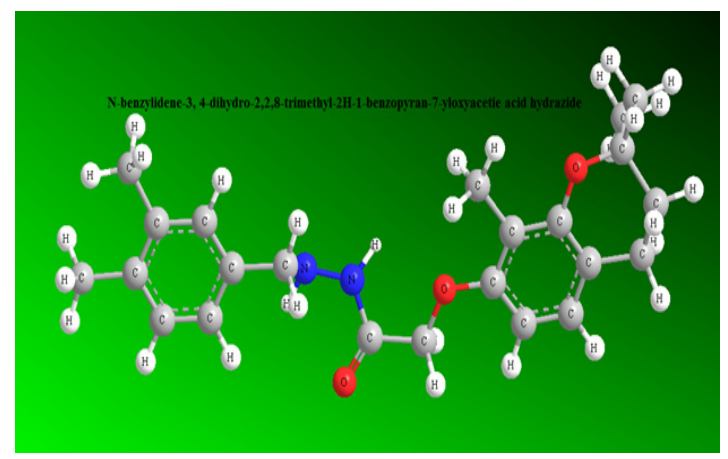

Fig. 7: Optimization of $\mathrm{N}$-benzylidene-3, 4-dihydro-2,2,8-trimethyl-2H-1-benzopyran-7yloxyacetie acid hydrazide

methyl 5-(2-propenoyl)-3- thiophene carboxylatein $60 \%$ yield. The proton $\mathrm{nmr}$ spectrum exhibit explicitly a doublets for two hydrogens due to the de-shielding effect of the carbonyl. Infra-red spectroscopy helps to confirm the structure of two carbonyls around 1650 $\mathrm{cm}^{-1}$ for the allylic ketone and $1700 \mathrm{~cm}^{-1}$ for the ester Figs1-3.

Since a carcinogen is applied into a body, cancerous cell will not immediately result. This is due to the "latency effect" where certain of time elapses before there is growth of the tumor. The initial application of a carcinogen will result in the formation of the irreversible initiated cells. Time may then elapse before a second agent, known as the promoter, will act reversibly on the initiated cell giving a premalignant lesion. Changes in the premalignant lesion, such as increased growth rate, increased invasiveness and metastases, result from the $3^{\text {rd }}$ stage of the process known as progression. Those changes are usually associated with the changing in the number and arrangement of genes which encode for various proteins.

\section{Theoretical background}

Aaromaticity in point of nucleus-independent chemical shifts, with NICS (0), at the center of ring plane were compared in several studies in long distances. In small range of distance a few works have been done in theoretical and reports the statistical approach in our works ${ }^{15}$

For anyfurther discussion of statistical methods in S-NICS especially in short range of distances, we exhibited that the asymmetry(ç) $)^{15}$ and $\operatorname{skew}(\hat{\mathrm{e}})^{15}$ fluctuate around the center of rings. 
The maximum fluctuations ${ }^{15}$ are visible around the extremums functions mathematically ${ }^{15}$.
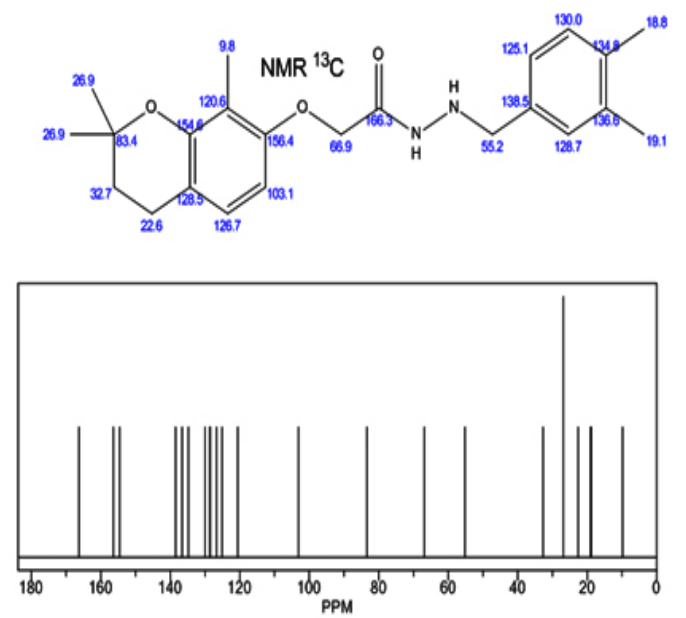

Fig. 8: Carbon NMR diagram of $\mathrm{N}$-benzylidene-3, 4-dihydro-2,2,8-trimethyl-2H1-benzopyran-7-yloxyacetie acid hydrazide
The fundamental of this work is based on random motions of dummy atom in de-shielding spaces of heterocyclic rings for considering the most abundant of points. The majour purpose of random data of several probes inside of de-shielding spaces is for understanding of anisotropic spin-spin interaction in short distances.

In this study, the major components ${ }^{16-18}$ of Haeberlen ${ }^{17}$ parameters ${ }^{16-18}$, has been calculated for heterocyclic rings. A large number of random points near to the center of those rings have been generated by pseudo-random numbers ${ }^{15}$ generation, which is distributed in a Gaussian function between the interval $[0,1)^{15}$.

The results have been compared through the energy-decomposition-analyzation (EDA). The $\pi$ bond energy and conjugation of between heteroatoms bonds of rings are signiûcantly accurate. We have optimized the geometries and calculated the carbon NMR for various heterocyclic molecules for understanding which members of rings are more stable Figs1-8. Our methods and physical chemistry approach have been done based on our previous works ${ }^{19-44}$.

Table 1: the principal data such as standard components, Haeberlen-Mehring (Isotropic and Anisotropy) for oxadiazol derivatives

\begin{tabular}{|c|c|c|c|c|c|c|c|}
\hline \multirow[t]{5}{*}{49} & $\mathrm{~Bq}$ & Isotropic & & 9.6670 & Anisotropy & & 5.0148 \\
\hline & $X X=$ & 9.4293 & $Y X=$ & 3.3067 & $Z X=$ & 1.0248 & \\
\hline & $X Y=$ & 1.9426 & $Y Y=$ & 9.7601 & $Z Y=$ & -2.9706 & \\
\hline & $X Z=$ & -0.0460 & $Y Z=$ & -1.8228 & $\mathrm{ZZ}=$ & 9.8115 & \\
\hline & Eigenvalues: & & & 5.8663 & 10.1245 & & 13.0102 \\
\hline \multirow[t]{5}{*}{50} & $\mathrm{~Bq}$ & Isotropic & & 8.0983 & Anisotropy & & 5.5104 \\
\hline & $X X=$ & 5.8732 & $Y X=$ & 2.2708 & $Z X=$ & 1.3457 & \\
\hline & $X Y=$ & 2.6023 & $Y Y=$ & 7.2310 & $Z Y=$ & 0.0328 & \\
\hline & $X Z=$ & 2.2751 & $Y Z=$ & -0.9279 & $Z Z=$ & 11.1908 & \\
\hline & Eigenvalues: & & & 3.6297 & 8.8933 & & 11.7720 \\
\hline \multirow[t]{5}{*}{51} & $\mathrm{~Bq}$ & Isotropic & & 10.3139 & Anisotropy & & 4.0072 \\
\hline & $X X=$ & 10.2125 & $Y X=$ & 2.4504 & $Z X=$ & 0.5820 & \\
\hline & $X Y=$ & 1.0666 & $Y Y=$ & 10.8610 & $Z Y=$ & -2.2914 & \\
\hline & $X Z=$ & -0.6321 & $Y Z=$ & -1.2240 & $\mathrm{ZZ}=$ & 9.8682 & \\
\hline & Eigenvalues: & & & 7.9371 & 10.0193 & & 12.9854 \\
\hline \multirow[t]{5}{*}{52} & $\mathrm{~Bq}$ & Isotropic & $=$ & 8.9431 & Anisotropy & $=$ & 3.8663 \\
\hline & $X X=$ & 9.8499 & $Y X=$ & 2.8955 & $Z X=$ & 1.4619 & \\
\hline & $X Y=$ & 2.1835 & $Y Y=$ & -2.0714 & $Z Z=$ & -2.2300 & \\
\hline & $X Z=$ & 0.2536 & $Y Z=$ & -2.0714 & $Z Z=$ & 9.9580 & \\
\hline & Eigenvalues: & & & 4.5560 & 10.7527 & & 11.5207 \\
\hline
\end{tabular}




\section{RESULTS AND DISCUSSION}

Optimization\& NMR constants with orientations of the principal components\& HaeberlenMehring ${ }^{17}$, or Herzfeld-Berger ${ }^{16-18}$ parameter for several heterocyclic compounds in random situations has been calculated through DFT methods tables1,2.

In small distance around the center, the asymmetric-parameter ${ }^{16}(\eta)$, and the $\operatorname{skew}^{17}(\kappa)$, exhibited. gaussian distribution based on their fluctuation behavior ${ }^{15}$, which is relate on its distance of molecular ring. In contrast, of that parameters, the isotropy ${ }^{16-18} \sigma_{\text {iso }}$ Has not a fluctuating behavior and increase in around the center of the rings with a linear relationship ${ }^{15}$. The slopes of that line is changed for various distances of heterocyclic compounds.

The isotropy ${ }^{16-18}$ in all NMR calculations are positive which indicates negative values for aromaticity ${ }^{15}$. It is obvious that the isotropies ${ }^{15}$ for NICS data can explain the quantity and quality of the aromaticity for some molecules, but those are not able for expressing the mechanism as well as S-NICS ${ }^{15}$.

In the S-NICS method ${ }^{15}$ the suitable shielding space near to the center of hetero rings are enables to evaluate the aromaticity ${ }^{15}$ as a criterion data. and in this method the expectation of the $\left(\eta^{\star}\right)^{16-}$ ${ }^{18}$ and $\left(\kappa^{*}\right)^{16-18}$ have been estimated via the Gaussian curve.

The isotropy $\left(\sigma_{\text {iso }}{ }^{*}\right)$ which is related to all of $\left(\eta^{\star}\right),\left(\kappa^{\star}\right),\left(\Omega^{\star}\right)$ and $\left(\zeta^{*}\right)$ is suitable criterion for the aromatic molecules both hetero or regular rings through the S-NICS method. Similar to the NICS method, in S-NICS ${ }^{15}$, mines nucleus-independentchemical-shifts indicates the aromaticity. Therefor "+" values indicate the anti-aromaticity quantitative. In S-NICS ${ }^{15}$ methods, the shielding\& de-shielding ${ }^{15}$ spaces are important for discussing the mechanism of the aromatic molecules in point of ring currents.

The stabilities S-NICS criterion is strongly affected on the best places in shielding spaces which is related to the composition of hetero aromatic rings. It is obvious that geometry factors cause changes in the magnetic-field ${ }^{15}$ by the nuclei and change the resonant frequencies. Therefore the chemical shielding and several factors as the same electronegativity, magnetic anisotropy of $\pi$-systems will be changed due to the number of electrons The chemical shielding is a vector orientation function for all of the shielding parameters that can change in several places inside the shielding region .

Table 2: S-NICS calculation for 14 center rings

\begin{tabular}{lccccc}
\hline $\begin{array}{l}\text { Dummy } \\
\text { atoms in } \\
\text { Rings } \\
\text { (number) }\end{array}$ & $\sigma_{11}$ & $\sigma_{22}$ & $\sigma_{33}$ & S-NICS & NICS \\
\hline & & & & & \\
BQ1 & 5.8663 & 10.1245 & 13.0102 & -9.667 & -9.122 \\
BQ2 & 3.6297 & 8.8933 & 11.7720 & -8.098 & -9.051 \\
BQ3 & 7.9371 & 10.0193 & 12.9854 & -10.313 & -9.265 \\
BQ4 & 4.5560 & 10.7527 & 11.5207 & -8.943 & -9.034 \\
BQ5 & 4.9321 & 9.3453 & 11.3456 & -8.541 & -9.01 \\
BQ6 & 5.0031 & 9.1267 & 10.9934 & -8.374 & -8.756 \\
BQ7 & 4.9835 & 9.3476 & 11.4523 & -8.594 & -8.765 \\
BQ8 & 5.1211 & 9.8745 & 10.9874 & -8.661 & -9.001 \\
BQ9 & 4.9135 & 11.84312 & 10.8734 & -9.211 & -9.324 \\
BQ10 & 6.6345 & 10.9833 & 11.9823 & -9.866 & -9.545 \\
BQ11 & 7.3462 & 11.0987 & 10.9872 & -9.811 & -9.934 \\
BQ12 & 4.9932 & 10.9453 & 11.9352 & -9.291 & -9.012 \\
BQ13 & 5.0923 & 10.9542 & 10.9987 & -9.015 & -9.241 \\
BQ14 & 6.9345 & 9.4581 & 11.0187 & -9.137 & -9.345 \\
\hline
\end{tabular}




\section{REFERENCES}

1. Gomtsyan, A. Chem. Heterocycl. Compd. 2012, 48,7-10.

2. Dua, R.; Shrivastava, S.; Sonwane, S.K.; Srivastava, S.K. Adv. Biol. Res. (Rennes). 2011, 5, 120-144.

3. Broughton, H.B.; Watson, I.A. J. Mol. Graph. Model.2004, 23, 51-58.

4. El-salam, N.M.A.; Mostafa, M.S.; Ahmed, G.A.; Alothman, O.Y. J. Chem. 2013, 2013, 1-8.

5. Azab, M.E.; Youssef, M.M.; El-Bordany, E.A. Molecules 2013, 18, 832-844.

6. Salem, M.S.; Sakr, S.I.; El-Senousy, W.M.; Madkour, H.M.F. Arch. Pharm. (Weinheim). 2013, 346, 766-773.

7. Cao, X.; Sun, Z.; Cao, Y.; Wang, R.; Cai, T.; Chu, W.; Hu, W.; Yang, Y. J. Med. Chem. 2014, 57, 3687-3706.

8. El-Sawy, E.R.; Ebaid, M.S.; Abo-Salem, H.M.; Al-Sehemi, A.G.; Mandour, A.H. Arab. J. Chem. 2013, 7, 914-923.

9. Chen, Y.; Yu, K.; Tan, N.Y.; Qiu, R.H.; Liu, W.; Luo, N.L.; Tong, L.; Au, C.T.; Luo, Z.Q.; Yin, S.F.Eur. J. Med. Chem. 2014, 79, 391-398.

10. El-Sawy, E.R.; Mandour, A.H.; El-Hallouty, S.M.; Shaker, K.H.; Abo-Salem, H.M. Arab. J. Chem. 2013, 6, 67-78.

11. Mabkhot, Y.N.; Barakat, A.; Al-Majid, A.M.; Alshahrani, S.; Yousuf, S.; Choudhary, M.I. Chem. Cent. J. 2013, 7, 112-120.

12. Vitaku, E.; Smith, D.T.; Njardarson, J.T. J. Med. Chem. 2014, 57, 10257-10274.

13. Verma, A.; Joshi, S.; Singh, D. J. Chem. 2013, $1-12$

14. D. S. Arora, Singh, J. and Aneja, K. R.Kluwer Academic/Plenum Publishers, New Yok,1999

15. Monajjemi, M.; Mohammadian, T Nayyer. J. Comput. Theor. Nanosci. 2015,12, 48954914

16. J. Herzfeld, A. E. Berger, J. Chem. Phys. 1980, 73, 6021

17. U. Haeberlen, In Advances in Magnetic Resonance, Suppl. 1 Academic Press, New York (1976), M. Mehring.M, Principles of High Resolution NMR in Solids, 2nd.ed, Springer Verlag, Berlin, H. W. Spiess, In NMR Basic
Principles and Progress; P. Diehl, E. Fluck, R. Kosfeld, Eds.; Springer Verlag, Berlin, 1978, 15.

18. D. P, Raleigh, F. Creuzet, S. K Das Gupta, M. H Levitt, and R. G Griffin. J. Am. Chem. Soc.1989, 111, 4502

19. Monajjemi, M.; Lee, V.S.; Khaleghian, M.; B. Honarparvar, B.; F. Mollaamin, F. J. Phys. Chem C. 2010, 114, 15315

20. Monajjemi, M, Journal of Molecular Liquids, 2017, 230 , 461-472

21. Jalilian,H.; Monajjemi, M. Japanese Journal of Applied Physics. 2015, 54(8), 08510

22. Monajjemi, M. Struct Chem. 2012, 23,551580

23. Monajjemi, M.; Boggs, J.E. J. Phys. Chem. $A$, 2013, 117, 1670 "1684

24. Mollaamin, F.; Monajjemi, M, Journal of Computational and Theoretical Nanoscience. 2012, 9 (4) 597-601

25. Monajjemi, M.; Khaleghian, M, Journal of Cluster Science. 2011, 22 (4), 673-692 318

26. Monajjemi, M.; Wayne Jr, Robert. Boggs, J.E. Chemical Physics. 2014, 433, 1-11

27. Monajjemi, M. Falahati, M.; Mollaamin, F.; Ionics, 2013, 19, 155-164

28. Monajjemi, M.; Mollaamin, F. Journal of Cluster Science, 2012, 23(2), 259-272

29. Tahan, A.; Monajjemi, M. Acta Biotheor, 2011, 59, 291-312

30. Mollaamin, F.; Monajjemi, M. Physics and Chemistry of Liquids .2012, 50(5) ,596-604

31. Monajjemi, M.; Khosravi, M.; Honarparvar, B.; Mollaamin, F.; International Journal of Quantum Chemistry, 2011, 111, 27712777

32. Monajjemi, M. Theor Chem Acc, 134:77 DOI 10.1007/s00214-015-1668-9 , 2015.

33. Monajjemi, M. Journal of Molecular Modeling , 2014, 20, 2507

34. Monajjemi, M.; Khaleghian, M.; Mollaamin, F. Molecular Simulation. 2010, 36(11), 865-

35. Monajjemi, M. Biophysical Chemistry. 2015, 207,114-127

36. Monajjemi, M.; Honaparvar, B.; Hadad, B. Khalili, African Journal of Pharmacy and Pharmacology , 2010, 4( 8),521-529 
37. Mollaamin, F.; Varmaghani, Z.; Monajjemi, M. Physics and Chemistry of Liquids 2011, 49( 3) $318-336$

38. Monajjemi, M.; Rajaeian, E.; Mollaamin, F, Physics and Chemistry of Liquids 2008, 46( 3) 299-306

39. Monajjemi, M.; Heshmat, M.; Aghaei, H.; Bulletin of the Chemical Society of Ethiopia, 21(1) 111-116

40. Monajjemi, M.; Aghaie, H.; Naderi, F. Biochemistry (Moscow) 2007, 72 ( 6 ), 652657

41. Monajjemi, M.; Honarparvar, B.; Nasseri, S. M. Journal of Structural Chemistry 2009, 50( 1) $67-77$
42. Mollaamin, F.; Baei, M. T.; Monajjemi, M.; Russian Journal of Physical Chemistry A, 2008, 82 (13), 2354-2361

43. Monajjemi, M.; Baei, M. T.; Mollaamin, F. Russian Journal of Inorganic Chemistry, 2008, 53 ( 9 ),1430-1437

44. Monajemi, M.; Ketabi, S.; Zadeh, MH; Biochemistry-Moscow 2006, 71, S1-S8

45. M. Monajjemi, Chemical Physics, 2013, 425, 29-45

46. M. Monajjemi, Macedonian Journal of Chemistry and Chemical Engineering, 2017, 36, 1, 101-118

47. M. Monajjemi, Journal of Molecular Liquids , 2017, 230 , 461-472 